\title{
Characterization of topological states on a lattice with Chern number
}

\author{
Mohammad Hafezi ${ }^{1}$, Anders S. Sørensen ${ }^{2}$, Mikhail D. Lukin ${ }^{1}$, Eugene Demler ${ }^{1}$ \\ 1 Physics department, Harvard university, Cambridge Massachusetts 02138 and \\ 2 QUANTOP, Danish National Research Foundation Centre of Quantum Optics, \\ Niels Bohr Institute, DK-2100 Copenhagen Ø, Denmark
}

\begin{abstract}
We study Chern numbers to characterize the ground state of strongly interacting systems on a lattice. This method allows us to perform a numerical characterization of bosonic fractional quantum Hall (FQH) states on a lattice where conventional overlap calculation with known continuum case such as Laughlin state, breaks down due to the lattice structure or dipole-dipole interaction. The non-vanishing Chern number indicates the existence of a topological order in the degenerate ground state manifold.
\end{abstract}

PACS numbers: 03.75.Lm,73.43.-f

One of the most dramatic manifestations of interactions in many-body systems is the appearance of new quantum states of matter. Often such states can be characterized by an order parameter and spontaneous breaking of some global symmetries. The 'smoking gun' evidence of such states is the appearance of Goldstone modes of spontaneously broken symmetries. However, some types of quantum many-body phases can not be characterized by a local order parameter. Examples can be found in FQH systems [1], lattice gauge theories 2], and spin liquid states 3 . Such states can be characterized by the topological order [4] which encompasses global geometrical properties such as ground state degeneracies on non-trivial manifolds [5]. Topologically ordered states often exhibit fractional excitations [6] and have been proposed as a basis of a new approach to quantum computations [7]. However, in many cases, identifying a topologically ordered state is a challenging task even for theoretical analysis. Given an exact wavefunction of the ground state in a finite system, how can one tell whether it describes a FQH phase of a $2 \mathrm{D}$ electron gas or a spin liquid phase on a lattice? One promising direction to identifying topological order is based on the Chern number calculations [8]. The idea of this approach is to relate the topological order to the geometrical phase of the many-body wavefunction under the change of the boundary conditions [9].

Important work of Berry [10] and Simon [11] initiated the investigation on geometrical phase factors and since then the field has been extensively studied in different contexts - for a review see, for example, [12]. In quantum Hall $(\mathrm{QH})$ systems, early works on the Chern number analysis [13] is focused on the Hall conductance and robustness of $\mathrm{QH}$ states against changes in the band structure [14] and the presence of disorder [5, 15]. Currently, there is also considerable interest in understanding FQH states in the presence of a strong periodic potential. Such systems are important in several contexts including anyonic spin states [16], vortex liquid states 17], and ultracold atoms in optical lattices 18, 19, 20, 21, 22] which are promising candidates for an experimental realization.

In this letter, we investigate a novel procedure for calculating Chern numbers and demonstrate that this method provides insight into the topological order of the ground state in regimes where other methods fails to provide a definite answer for the nature of the ground state wavefunction. In particular, we study a fractional quantum Hall system with bosons on a lattice with a filling factor of $\nu=1 / 2$, where $\nu$ is the ratio of the number of magnetic flux quanta to the number of particles. In the continuum limit, where the flux-fraction through each plaquette $\alpha$ is very small $(\alpha \ll 1)$, this system is exactly described by the Laughlin wavefunction. However, in a recent study 18], it was found that for some values of $\alpha \gtrsim 0.25$, the Laughlin wavefunction ceases to be a good description of the system, indicated by a decreasing overlap between the ground state and the Laughlin wavefunction. From this study, it is unclear whether this represented a change in the nature of the ground state, or just that the lattice structure distorts the state. Here, we use the Chern number calculation to provide an unambiguous characterization of the ground state even outside the regime where there is a significant overlap with the Laughlin wavefunction. In particular, we show that the Chern number and hence the topological order of the system remains undisturbed until $\alpha \lesssim 0$.4.(Tab【).

To introduce the basic ideas of the Chern number calculation in many-body system, we first discuss the continuum regime. We consider a single particle with charge $\mathbf{q}$ on a torus $T^{2}\left(L_{x} \times L_{y}\right)$ in the presence of a magnetic field $\mathbf{B}$ perpendicular to the surface. The corresponding Hamiltonian is invariant under the magnetic translation of single particle $\mathbf{s}, t_{s}(\mathbf{a})=e^{i \mathbf{a} \cdot k^{s} / \hbar}$ where $\mathbf{a}$ is a vector on the torus, and $k^{s}$ is the pseudo-momentum of particle $\mathbf{s}$, defined by $k_{x(y)}=-i \hbar \frac{\partial}{\partial x(y)}-q A_{x(y)} \mp q B y(x)$ in $\mathrm{x}$ and (y) direction, respectively, and $\vec{A}$ is the corresponding vector potential. The generalized boundary condition is given by the translation, $t_{s}\left(L_{i} \hat{i}\right) \psi\left(x_{s}, y_{s}\right)=e^{i \theta_{i}} \psi\left(x_{s}, y_{s}\right)$, where $(i=1,2)$ refer to two directions $(\mathrm{x}, \mathrm{y})$ on the torus $T^{2}$ and the $\theta_{i}$ 's are twist angles of the boundary (Fig[1 a). The magnetic phase through each plaquette $(2 \pi \alpha)$ arises from the field perpendicular to the surface of the torus. The Chern number for non-degenerate state $\alpha$ is defined by, 

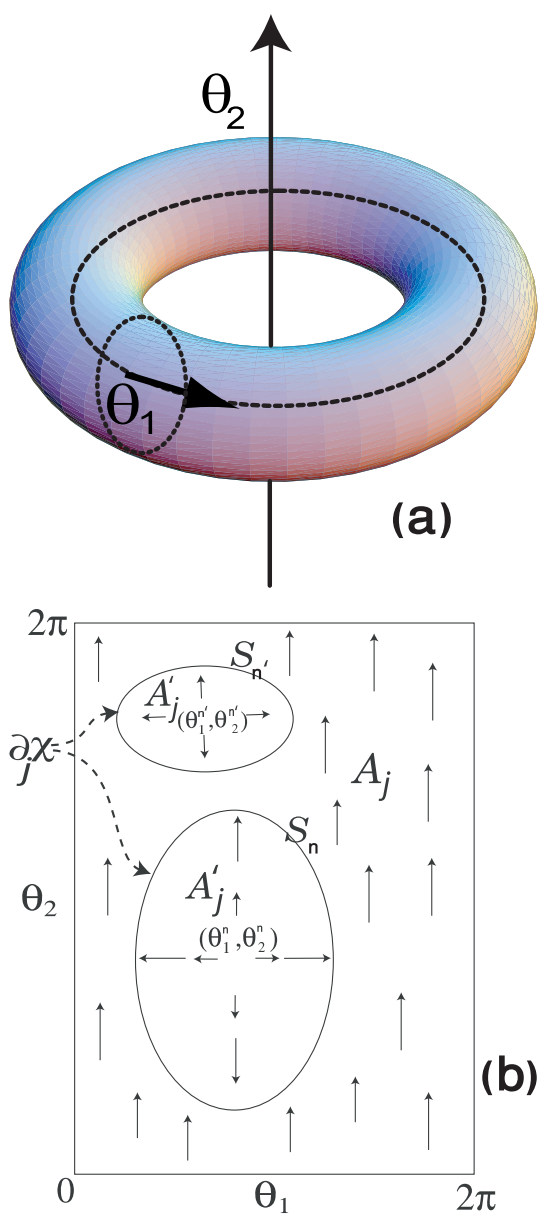

FIG. 1: (a) Twist angles of the boundary condition as the result of two magnetic fluxes threaded the torus (b) Redefining the vector potential around the singularities: $\mathcal{A}_{j}$ is not welldefined everywhere on the torus of the boundary condition. Therefore, another vector field $\mathcal{A}^{\prime}{ }_{j}$ with different definition should be introduced around each singularity $\left(\theta_{1}^{n}, \theta_{2}^{n}\right)$ of $\mathcal{A}_{j}$. However, $\mathcal{A}_{j}$ and $\mathcal{A}^{\prime}{ }_{j}$ are related to each other with a gauge transformation $\chi$ and the Chern number depends only on the loop integrals of $\chi$ around those singularity regions.

$$
C(\alpha)=\frac{1}{2 \pi} \int_{0}^{2 \pi} d \theta_{1} \int_{0}^{2 \pi} d \theta_{2}\left(\partial_{1} \mathcal{A}_{2}^{(\alpha)}-\partial_{2} \mathcal{A}_{1}^{(\alpha)}\right)
$$

where $\mathcal{A}_{j}^{(\alpha)}\left(\theta_{1}, \theta_{2}\right)$ is defined as a vector field based on the eigenstate $\Psi^{(\alpha)}\left(\theta_{1}, \theta_{2}\right)$ on $T^{2}$ by $\mathcal{A}_{j}^{(\alpha)}\left(\theta_{1}, \theta_{2}\right) \doteq$ $i\left\langle\Psi^{(\alpha)}\left|\frac{\partial}{\partial \theta_{j}}\right| \Psi^{(\alpha)}\right\rangle$.

In the context of QH systems, the time derivative of twist angles could be considered as voltage drops across the Hall device in two dimensions and the boundary averaged Hall conductance of the any state is related to the Chern number of that state $[9]: \sigma_{H}^{\alpha}=C(\alpha) e^{2} / h$.

The non-trivial behavior (non-zero conductance in the case of quantum Hall system) occurs because of singularities of the vector field. If for a given non-degenerate state the corresponding vector field is not defined for certain angles $\left(\theta_{1}^{n}, \theta_{2}^{n}\right)$ in $S_{n}$ regions (Fig 10), then we should introduce a new well-defined vector field $\mathcal{A}_{j}^{\prime(\alpha)}\left(\theta_{1}, \theta_{2}\right)$, inside those regions. These two vector fields differ from each other by a gauge transformation, $\mathcal{A}_{j}^{(\alpha)}\left(\theta_{1}, \theta_{2}\right)-$ $\mathcal{A}_{j}^{\prime(\alpha)}\left(\theta_{1}, \theta_{2}\right)=\partial_{j} \chi\left(\theta_{1}, \theta_{2}\right)$ and the Chern number reduces to the winding number of the gauge transformation $\chi\left(\theta_{1}, \theta_{2}\right)$ over small loops encircling $\left(\theta_{1}^{n}, \theta_{2}^{n}\right)$, i.e. $\partial S_{n}$ [13],

$$
C(\alpha)=\sum_{n} \frac{1}{2 \pi} \oint_{\partial S_{n}} \vec{\nabla} \chi \cdot d \vec{\theta} .
$$

For the case of degenerate ground state a generalization of the above argument can be made, where instead of having a single vector field $\mathcal{A}_{j}^{(\alpha)}\left(\theta_{1}, \theta_{2}\right)$, a tensor field $\mathcal{A}_{j}^{(\alpha, \beta)}\left(\theta_{1}, \theta_{2}\right)$ should be defined, $\alpha, \beta=1 \ldots q$ for a $q$-fold degenerate ground state: $\mathcal{A}_{j}^{(\alpha, \beta)}\left(\theta_{1}, \theta_{2}\right) \doteq$ $i\left\langle\Psi^{(\alpha)}\left|\frac{\partial}{\partial \theta_{j}}\right| \Psi^{(\beta)}\right\rangle$.

Therefore, when $\mathcal{A}_{j}^{(\alpha, \beta)}$ is not defined, similar to the non-degenerate case, a new gauge convention should be acquired for those regions with singularities. This gives rise to a tensor gauge transformation on the border of these regions, $\partial_{j} \chi^{(\alpha, \beta)}\left(\theta_{1}, \theta_{2}\right)=\mathcal{A}_{j}^{(\alpha, \beta)}\left(\theta_{1}, \theta_{2}\right)-$ $\mathcal{A}_{j}^{\prime(\alpha, \beta)}\left(\theta_{1}, \theta_{2}\right)$ and consequently the Chern number is given by the trace of the tensor $\chi$,

$$
C(1,2, \ldots, q)=\sum_{n} \frac{1}{2 \pi} \oint_{\partial S_{n}} \vec{\nabla} \operatorname{Tr} \chi^{(\alpha, \beta)} \cdot d \vec{\theta}
$$

We focus on a system of bosons on a square lattice described by the Hamiltonian [19]:

$$
\begin{aligned}
H & =-J \sum_{x, y} \hat{a}_{x+1, y}^{\dagger} \hat{a}_{x, y} e^{-i \pi \alpha y}+\hat{a}_{x, y+1}^{\dagger} \hat{a}_{x, y} e^{i \pi \alpha x}+\text { h.c. } \\
& +U \sum_{x, y} \hat{n}_{x, y}\left(\hat{n}_{x, y}-1\right)
\end{aligned}
$$

where $J$ is the hopping energy between two neighboring sites, $U$ is the on-site interaction energy, and $2 \pi \alpha$ is the phase acquired by a particle going around a plaquette. We concentrate on the hardcore limit $(U \gg J)$ and $\nu=1 / 2$ where $\nu$ is the ratio of the number of particles to the total number of flux in the system. The experimental proposal for realizing such a Hamiltonian for atomic gases confined in an optical lattice has already been investigated [18, 19]. The ground state of the system for very dilute lattice $\alpha \lesssim 0.2$ is two-fold degenerate and is well described by Laughlin state. When $\alpha$ increases the lattice structure becomes more apparent and the overlap with Laughlin wavefunction breaks down. However, by numerical calculation, we show that Chern number characterizes system better and remains the same, i.e. 1/2 

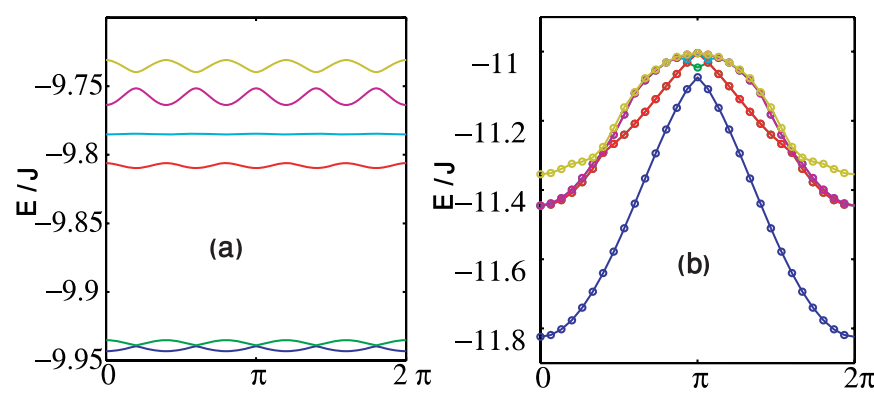

FIG. 2: Low-lying energy levels as a function of twist angles. For finite $\alpha$ the ground state energy oscillates as a function of twist angles and for high $\alpha \gtrsim 0.4$ the oscillations reach the exited levels. (a) shows lowest energy levels for $\alpha=0.32$ (4 atoms on a $5 \times 5$ lattice) while (b) shows the five lowest energy levels for $\alpha=0.4$ ( 5 atoms on a $5 \times 5$ lattice) In both plots, $\theta_{2}=\pi$ and $\theta_{1}$ is varied from zero to $2 \pi$.

for each state in the ground state manifold, for systems with higher flux density $\alpha \lesssim 0.4$.

In the case of a very dilute lattice $\alpha \ll 1$, i.e. the magnetic length is much larger than the lattice spacing, and hence the lattice Hamiltonian approaches the continuum limit. According to Haldane 23], the magnetic translational symmetry of the center of mass results in a two-fold degeneracy of the ground state for $\nu=1 / 2$. However, by increasing the magnetic field, the lattice structure becomes more pronounced, in such a way that even for a single particle, the lattice modifies the energy levels from being simple Landau levels into the fractal structure known as the Hofstadter butterfly [24]. For the many-body problem, the presence of the lattice causes the energy levels to oscillate (see Fig. 2 2 a) and instead of having a unique degeneracy for all twist angles values, the ground state is two-fold degenerate at only certain twist angles. However, the two-dimensional ground state manifold is well defined and separated from the other states. By integrating over twist angles, one state mixes with the other state when levels touch each other, therefore in the Chern number evaluation, both levels participate and the degenerate form of the Chern number should be used (Eq. 3).

It is important to note that the degeneracy in the noninteracting regime (Landau degeneracy) is fundamentally different from that of the interacting hard-core case. In the non-interacting limit $(U \ll J)$, the degeneracy can be lifted by a local perturbation e.g. an impurity, while in the hardcore case, the degeneracy remains in the thermodynamic limit [5]. The latter degeneracy is a consequence of the global non-trivial properties of the manifold on which the particles move rather than symmetries of the Hamiltonian (e.g. the Ising model) [4]. Recently, it was shown [6] that in presence of a gap, there is a direct connection between the fractionalization and the topological degeneracy. In particular, the amount of the degeneracy is related to the statistics of the fractionalized quasipar- ticles e.g. in the case of $\nu=1 / 2$, the two-fold degeneracy is related to $1 / 2$ anyonic statistics of the corresponding quasiparticles.

The ground state degeneracy prevents the direct integration of Eq. (1) since wavefunctions would mix together when twist angles vary. Therefore, one has to use Eq. (3) and also resolve the extra gauge related to the ground state. We can consider two possibilities: fixing the relative phase between the two states in the ground state, or lifting the degeneracy by adding some impurities. In the latter case, we can show that the system has a topological order in spite of poor overlap with the Laughlin state [19]. On the other hand, a significant amount of impurity in the system may distort the energy spectrum, so that the underlying physical properties of the lattice and fluxes could be confounded by the artifacts due to the impurities, especially for large $\alpha$. Therefore, in this letter we focus on the degenerate case.

We start with the simple case of a non-degenerate ground state on a discrete s-dimensional Hilbert space, $\Psi\left(\theta_{1}, \theta_{2}\right)=\left(c_{1}, c_{2}, \ldots, c_{s}\right)$. The one-dimensional gauge can be resolved by making two conventions: in one convention the first element and in the other the second element of the wavefunction in the Hilbert space should be real i.e. we transform the ground state $\Psi$ into $\Psi_{\Phi}=P \Phi=\Psi \Psi^{\dagger} \Phi$ where $\Phi=(1,0, \ldots, 0)^{\dagger}$ is a s-dimensional vector and $P$ is a projection into the ground state and similarly with the other reference vector $\Phi^{\prime}=(0,1, \ldots, 0)^{\dagger}$. Hence, we can uniquely determine the gauge $\chi$ which relates the two corresponding vector fields: $e^{i \chi}=\Phi^{\dagger} P \Phi^{\prime}$. Therefore, the Chern number will be equal to the number of vorticities of $\chi$ around regions where $\Lambda_{\phi}=\Phi^{\dagger} P \Phi=\left|c_{1}\right|^{2}$ is zero.

For fixing the q-dimensional ground state manifold gauge, we take two reference multiplets $\Phi$ and $\Phi^{\prime}$ which are two $\mathbf{s} \times \mathbf{q}$ matrices $(\mathbf{q}=\mathbf{2}$ in our case). We define an overlap matrix as $\Lambda_{\phi}=\Phi^{\dagger} P \Phi$, and consider the regions where $\operatorname{det} \Lambda_{\Phi}$ or $\operatorname{det} \Lambda_{\Phi}^{\prime}$ vanishes (similar to zeros of the wave function in the non-degenerate case). Hence, the Chern number for $\mathbf{q}$ degenerate states will be equal to the total winding number of $\operatorname{Tr} \chi^{(\alpha, \beta)}$ for small neighborhoods $S_{n}$, in which $\operatorname{det} \Lambda_{\Phi}$ vanishes. It should be noted that the zeros of $\operatorname{det} \Lambda_{\Phi}$ and $\operatorname{det} \Lambda_{\Phi}^{\prime}$ should not coincide in order to uniquely determine the total vorticity. In our numerical calculation, we choose multiplets $\Phi$ and $\Phi^{\prime}$ to be two sets of two degenerate ground states at two different twist angles far apart e.g. $(0,0)$ and $(\pi, \pi)$. In Fig. 3, we have plotted $\Omega=\operatorname{det}\left(\Phi^{\dagger} P \Phi^{\prime}\right), \operatorname{det} \Lambda_{\Phi}$, and $\operatorname{det} \Lambda_{\Phi}^{\prime}$, found by numerical diagonalization of the Hamiltonian over a grid $(30 \times 30)$ of twist angles $\theta_{1}$ and $\theta_{2}$. The Chern number can be determined by counting the number of vortices and it is readily seen that the winding number is equal to one for the corresponding zeros of $\operatorname{det} \Lambda_{\Phi}^{\prime}$ and $\operatorname{det} \Lambda_{\Phi}$.

We have calculated the Chern number for fixed $\nu=1 / 2$ and different $\alpha$ 's by the method described above. The result is shown in Tab! For $\alpha \ll 1$, we know from previous calculation [18] that the ground state is the Laughlin 

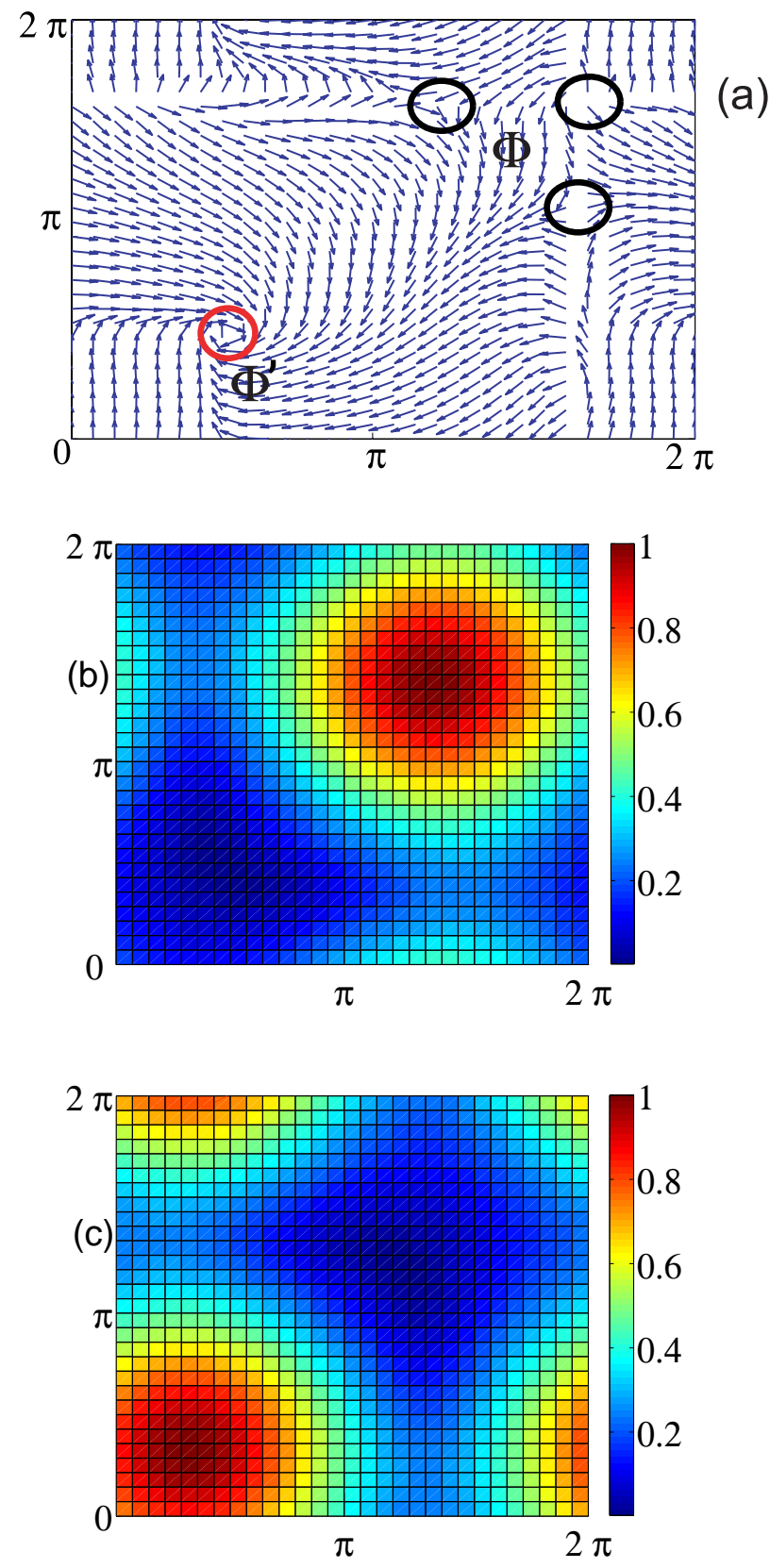

FIG. 3: (a) $\Omega\left(\theta_{1}, \theta_{2}\right)$ for fixed $\Phi$ and $\Phi^{\prime}$. $\theta_{1}$ and $\theta_{2}$ changes form zero to $2 \pi$. This plot has been produced for 4 atoms in the hard-core limit on a $5 \times 5$ lattice $(\alpha=0.32)$. (b) and (c): surface plots of $\operatorname{det} \Lambda_{\Phi}$ and $\operatorname{det} \Lambda_{\Phi}^{\prime}$ (blue is lower than red). $\theta_{1}$ and $\theta_{2}$ changes form zero to $2 \pi$. The total vorticity corresponding to each of trial function ( $\Phi$ or $\Phi^{\prime}$ ) indicates a Chern number equal to one for the two dimensional ground state manifold.

state and we expect to obtain a Chern number equal to $1 / 2$ for each state i.e. total Chern number equal to one. For higher $\alpha$, the lattice structure becomes more apparent and the overlap with the Laughlin state decreases rapidly. However, in our calculation, the ground state remains two-fold degenerate and the associated Chern

\begin{tabular}{|c|c|c|c|c|c|}
\hline Atoms & Lattice & $\alpha$ & gap /J & Chern/state & Overlap \\
\hline 3 & $6 \times 6$ & .17 & 0.24 & $1 / 2$ & 0.99 \\
\hline 4 & $6 \times 6$ & .22 & 0.24 & $1 / 2$ & 0.98 \\
\hline 3 & $5 \times 5$ & .24 & 0.23 & $1 / 2$ & 0.98 \\
\hline 3 & $4 \times 5$ & .3 & 0.18 & $1 / 2$ & 0.91 \\
\hline 4 & $5 \times 5$ & .32 & 0.15 & $1 / 2$ & 0.78 \\
\hline 3 & $4 \times 4$ & .375 & 0.03 & $1 / 2$ & 0.29 \\
\hline
\end{tabular}

TABLE I: Chern Number for different configurations in the hard-core limit for fixed filling factor $\nu=1 / 2$. The Laughlin state overlap is shown in the last column. although it deviates from the Laughlin state. Although the ground state deviates from the Laughlin state, the Chern number remains equal to one half per state before reaching some critical $\alpha_{c} \simeq 0.4$ where the energy gap vanishes.

number remains equal to one before reaching some critical $\alpha_{c} \simeq 0.4$. Hence, we expect to have similar topological order and fractional statistics of the excitations on the lattice in this regime.

For higher flux densities, $\alpha>\alpha_{c}$, the two-fold ground state degeneracy is no longer valid everywhere on the torus of the boundary condition. In this regime, the issue of degeneracy is more subtle, and finite size effect becomes significant. The translational symmetry argument [23] is no longer valid and the degeneracy of the ground state varies periodically with the system size [25]. Some gaps might be due to the finite size and vanish in the thermodynamic limit. To investigate this, we study the ground state degeneracy as a function of boundary angles $\left(\theta_{1}, \theta_{2}\right)$ which are not physical observable. Therefore, the degeneracy in thermodynamic limit should not depend on the their value. In particular, Fig. 2b shows the energy levels of five particles at $\alpha=0.4$ for different values of twist angles. The first and the second level are split at $\left(\theta_{1}=\theta_{2}=0\right)$, while they touch each other at $\left(\theta_{1}=\theta_{2}=\pi\right)$. We have observed similar behavior for different number of particles and lattice sizes e.g. 3 and 4 atoms at $\alpha=0.5$. Therefore, the ground state enters a different regime which is a subject for further investigation. Existence of the topological order does not require a very strong interaction i.e. hard-core limit. Even at finite interaction strength $U \sim J \alpha$, we have observed the same topological order with the help of the Chern number calculation. If $U$ gets further smaller, the energy gap above the ground state diminishes [19] and the topological order disappears.

One of the impediment of the experimental realization of Quantum Hall state is the smallness of the gap which can be improved in presence of the dipole-dipole interaction [19]. The dipole interaction can be represented as extra term $\sum_{i j} U_{d} \mathbf{n}_{\mathbf{i}} \mathbf{n}_{\mathbf{j}} /\left|\mathbf{r}_{\mathbf{i}}-\mathbf{r}_{\mathbf{j}}\right|^{3}$ in the Hamiltonian Eq. (4), where $n_{i}$ is the number of particles at location $\mathbf{r}_{\mathbf{i}}$ in the units of lattice spacing and $U_{d}$ is the strength of the interaction. The magnetic dipole-dipole interaction has been achieved in Bose-Einstein condensation of 
Chromium [26], however, for a lattice realization, polar molecules with strong permanent electric dipole moments are more promising candidates, where the dipole interaction can be an order of magnitude greater than the tunneling energy. In the presence of such strong long-range interaction, the ground state deviates from the conventional FQH state even in the continuum case (i.e. for even $\alpha<0.2$, the overlap with the Laughlin wavefunction decreases by increasing the strength of the dipole interaction). However, by evaluating Chern number, we are able to identify the topological order of the system, that turns out to be intact i.e. Chern number equal to one for the two-fold degenerate ground state.

In conclusion, we have investigated a method to unambiguously calculate the Chern number for the ground state of a system. For the FQHE system on a lattice that we have investigated, the Laughlin wavefunction ceases to be a good description of the ground state for high fluxes $\alpha \gtrsim 0.25$, but the Chern number remains $1 / 2$ per state until $\alpha \lesssim 0.4$ which is a direct indication of topological order in the system. Calculating Chern numbers by this method can be generalized for finite lattice systems to properly characterize the ground state manifold which is otherwise impossible with conventional overlap methods.

We thank K. Yang and S. Girvin for fruitful discussions. This work was partially supported by the NSF Career award, Packard Foundation, AFSOR and the Danish Natural Science Research Council.
[1] S. M. Girvin et al., Phys. Rev. Lett. 58, 1252 (1987).

[2] P. A. Lee et al., Rev. Mod. Phys. 78, 17 (2006).

[3] P. W. Anderson, Science 235, 1196 (1987).

[4] X.-G. Wen, Adv. Phys. 44, 405 (1995).

[5] X. G. Wen and Q. Niu, Phys. Rev. B 41, 9377 (1990).

[6] M. Oshikawa et al., Phys. Rev. Lett. 96, 060601 (2006).

[7] A. Y. Kitaev, Annals. Phys. 303, 2 (2003).

[8] Y. Hatsugai, J. Phys. Jpn. 74, 1374 (2005).

[9] Q. Niu et al., Phys. Rev. B 31, 3372 (1985).

[10] M. V. Berry, Proc. R. Soc. A 382, 45 (1984).

[11] B. Simon, Phys. Rev. Lett. 51, 2167 (1983).

[12] A. Bohm et al., The Geometric Phase in Quantum Systems (Springer, 2003).

[13] M. Kohmoto, Ann. Phys. (N.Y.) 160, 343 (1985).

[14] D. J. Thouless et al., Phys. Rev. Lett 49, 405 (1982).
[15] D. N. Sheng et al., Phys. Rev. Lett. 90, 256802 (2003).

[16] A. Kitaev, Ann. Phys. (N.Y.) 321, 2 (2005).

[17] L. Balents et al., Phys. Rev. B 71, 144508 (2005).

[18] A. Sørensen et al., Phys. Rev. Lett. 94, 086803 (2005).

[19] M. Hafezi et al., Phys. Rev. A. 76 (2007).

[20] R. Palmer et al., Phys. Rev. Lett. 96, 180407 (2006).

[21] E. J. Mueller, Phys. Rev. A 70, 041603 (2004).

[22] D. Jaksch and P. Zoller, New J. Phys. 5, Art.56 (2003).

[23] F. Haldane, Phys. Rev. Lett. 55, 2095 (1985).

[24] D. Hofstadter, Phys. Rev. B 14, 2239 (1976).

[25] A. Kol and N. Read, Phys. Rev. B 48, 8890 (1993).

[26] A. Griesmaier, J. Werner, S. Hensler, J. Stuhler, and T. Pfau, Phys. Rev. Lett. 94, 160401 (2005). 